MVZ vor. Da Krankenhäuser von staatlichen, kommunalen, freigemeinnützigen und privaten Trägern betrieben werden, kann nicht ausgeschlossen werden, dass Kapitalinvestoren über den Erwerb eines Krankenhauses ein MVZ gründen und so auch an der vertragsärztlichen Versorgung beteiligt sein können. Das ist indessen eine unvermeidliche Folge der Trägervielfalt im Krankenhausbereich und stellt die Berechtigung des Gesetzgebers zu einer Differenzierung zwischen Krankenhäusern und anderen Leistungserbringern hinsichtlich der Gründereigenschaft nicht in Frage. [...]

https://doi.org/10.1007/s00350-019-5196-9

\section{Anmerkung zu BSG, Urt. v. 16.5.2018 - B 6 KA 1/17 (Hessisches LSG) Marius G. Hoßbach}

Das BSG hat im Mai 2018 die seit längerem umstritten Frage, ob MVZ selbst weitere MVZ gründen dürfen, abschlägig entschieden. Das Gericht führt zur Begründung zunächst an, dass sich eine Gründungsberechtigung für MVZ nicht positiv aus dem Gesetz ergibt (Rdnrn. 22 und 23). Zahnärzte und Psychotherapeuten sind in $\$ 95$ Abs. 1a SGB V jedoch auch nicht genannt, obwohl sie zweifellos und nach allgemeiner Auffassung gründungsberechtigt sind. Es wird mithin deutlich, dass der Gesetzgeber des GKV-VStG schlicht an die Konstellation nicht gedacht hat, dass MVZ selbst als MVZ-Gründer in Betracht kommen können. Andernfalls hätte der Gesetzgeber das MVZ entweder in die Aufzählung aufgenommen, explizit ausgeschlossen, die Aufzählung durch die Aufnahme des Terminus ,,insbesondere“ als nicht abschließend deklariert oder zumindest seine Entscheidung in der Frage in der Gesetzesbegründung dargelegt. Folglich ist es müßig und wenig zielführend, auf die konkrete Absicht des Gesetzgebers in der Frage abzustellen oder nach Anhaltspunkten dafür zu suchen, da eine gesetzgeberische Meinungsbildung dazu allem Anschein nach ausfiel ${ }^{1}$. Jedenfalls kann es vor dem Hintergrund, dass z.B. auch Zahnärzte in $\$ 95$ Abs. 1a SGB V nicht genannt werden, nicht ausschlaggebend sein, darauf hinzuweisen, dass der Katalog abschließend sei (vgl. BSG, Rdnrn. 28 und 30).

Ferner überzeugt der Hinweis des Gerichts wenig, dass „Gemeinschaften von Leistungserbringern“ in $\$ 95$ Abs. 1 S. 3 SGB V a.F. nicht erwähnt waren und damit ,,insbesondere Kassenärztliche Vereinigungen und Gemeinschaftspraxen ausgeschlossen“" seien (Rdnr. 27). Denn als Gemeinschaft von Leistungserbringern kommen allenfalls Praxisgemeinschaften in Betracht. Gemeinschaftspraxen, also Berufsausübungsgemeinschaften, und eben MVZ sind hingegen selbst Leistungserbringer mit eigener Abrechnungsnummer. Es ist zudem dem Zulassungswesen auch nicht fremd, dass auch Leistungserbringer, die sich aus einzelnen Gründungsberechtigen zu einer Kooperation zusammengeschlossen haben, ihrerseits zu einer weiteren Kooperation zusammentun, z. B. einer Praxisgemeinschaft. Schließlich gilt auch hier, dass von einer (Nicht -) Erwähnung im Gesetz kaum auf eine bewusste Entscheidung des Gesetzgebers gefolgert werden kann.

Weiterhin argumentiert das BSG hinsichtlich einer systematischen Auslegung damit, dass sich die Gründungsberechtigung für MVZ auch nicht aus $\$ 72$ Abs. 1 S. 2 SGB V ergeben würde, da die entsprechende Anwendung nicht nur dann ausgeschlossen ist, wenn etwas Abweichendes

Dr. iur. Marius G. Hoßbach,

K+ Korten Rechtsanwälte AG,

Neuer Wall 44, 20354 Hamburg, Deutschland bestimmt ist, sondern auch, ,wenn sich aus dem systematischen Zusammenhang der maßgeblichen Vorschriften und dem Wesen der jeweiligen Rechtsmaterie ergibt, dass eine entsprechende Anwendung nicht in Betracht kommt, etwa weil dem die Grundstruktur des Medizinischen Versorgungszentrums entgegensteht" ${ }^{2}$. Das darf allerdings nur mit der Einschränkung gelten, dass sich aus dem Kontext der jeweiligen Regelung eindeutig ergibt, dass eine entsprechende Anwendung offensichtlich nicht in Frage kommt. Ohne eine derartige Evidenz besteht die Gefahr, dass eine entsprechende Anwendung gemäß \$72 Abs. 1 S. 2 SGB V contra legem ausgeschlossen wird. Der Hinweis des Gerichts, dass es widersprüchlich erscheine, eine Bestimmung zur Entstehung eines MVZ auf ein MVZ entsprechend anzuwenden (Rdnr. 30), ist unterdessen einleuchtend und kann dazu führen, dass vorliegend die „Evidenz-Hürde“ überschritten wird.

Insgesamt wäre es indes wünschenswert gewesen, wenn sich das BSG vor dem Hintergrund, dass der Gesetzgeber das Interesse der Gründung von MVZ durch MVZ selbst nicht berücksichtigt hat, mehr auf die gesetzgeberischen Intentionen im Gesamtbild eingegangen wäre, die bei dem GMG von 2003 und dem GKV-VStG von 2011 zum Ausdruck gekommen sind $^{3}$. Rein systematische Erwägungen dürften folglich in dieser Rechtsfrage nicht den Ausschlag geben.

Dem Gesetzgeber war im Wesentlichen daran gelegen, die Unabhängigkeit medizinischer Entscheidungen in der vertragsärztlichen Versorgung vor reinen Kapitalinteressen $\mathrm{zu}$ schützen und um die Verhinderung eines Mittelabfluss an private, rein gewinnorientierte Organisationen ${ }^{4}$. Außerdem wollte der Gesetzgeber verhindern, dass ausschließlich kapitalmotivierte Investoren über MVZ und deren Trägergesellschaften immer mehr Zulassungen erwerben, sodass für freiberufliche Ärzte immer weniger Vertragsarztsitze zur Verfügung stehen ${ }^{5}$. Das BSG geht darauf teilweise ein und weist darauf hin, dass es dem Gesetzgeber darum ging, den Gründerkreis der MVZ eher zu beschränken als auszudehnen (Rdnr. 30). Dass Kommunen 2015 mit dem GKV-VSG ${ }^{6}$ in den Kreis der Gründungsberechtigten aufgenommen wurden, darf zu Recht als Ausnahme dazu verstanden werden. Eine eingehende Auseinandersetzung mit der Frage, ob MVZ-Gründungen durch MVZ mit den drei postulierten Motivationen des Gesetzgebers - ,Unabhängigkeit medizinischer Entscheidungen', ,drohender Mittelabfluss" und ,weniger Vertragsarztsitze für Freiberufler vereinbar sind, wäre mithin wünschenswert gewesen, denn tatsächlich bestehen keine konkreten Hinweise darauf, dass eine Gründungsberechtigung von MVZ diesen Leitgedanken des Gesetzgebers entgegenstehen würde ${ }^{7}$.

Die wirtschaftliche Bedeutung der Entscheidung dürfte schließlich überschaubar sein. Es gibt schätzungsweise ca. 300 solcher „Alt-MVZ“8. Wie viele davon wiederum weitere MVZ gründen wollten, ist nicht ermittelt. Mithin gewährt der umfassende Bestandsschutz gem. \95 Abs. 1a S. 2 SGB V den MVZ, weitere Zweigstellen zu gründen und weitere Arztsitze zu erwerben, sodass auch eine wirtschaftliche Expansion weiterhin möglich ist.

1) Vgl. BT-Dr. 17/6906, S. 70 f.

2) So bereits BSGE 100, $144=$ SozR 4-2500 \$85 Nr. 41 (Rdnr. 18); BSG, SozR 4-2500 \$121 Nr. 7 (Rdnr. 12); BSG, SozR 4-2500 $\$ 103$ Nr. 7 (Rdnr. 23).

3) Vgl. BT-Dr. 15/1525, S. 108, BT-Dr. 17/6906, S. 70.

4) BT-Dr. 17/6906, S. 70.

5) BT-Dr. 17/6906, S. 70.

6) GKV-Versorgungsstärkungsgesetz v. 16.7.2015, BGBl. I S. 1211.

7) Darauf eingehend bereits die Anmerkung des Verfassers zur Vorinstanz, LSG Hess., Urt. v. 30.11.2016 - L 4 KA 20/14, MedR 2017, $905 \mathrm{f}$.

8) Die Schätzung teilte die Geschäftsführung des BMVZ dem Verfasser auf Anfrage mit Stellungnahme v. 25.7.2017 mit. 